\title{
Phase contrast mri measurement of e/a and e/e'
}

\author{
Neil Chatterjee ${ }^{1 *}$, Jeremy Collins ${ }^{2}$, James Carr ${ }^{2}$, Peter J Weale ${ }^{3}$ \\ From 2011 SCMR/Euro CMR Joint Scientific Sessions \\ Nice, France. 3-6 February 2011
}

\section{Introduction}

MRI is the accepted gold standard for assessment of left ventricular systolic function; however, no standards are available to assess diastolic function at MRI. E/A and E/ e' ratios are currently used in echocardiography to evaluate left ventricular diastolic function. Measuring these ratios with phase contrast MRI may provide a complementary approach to assessing left ventricular function.

\section{Purpose}

To validate E/A and E/e' ratios acquired with phase contrast MRI relative to established values using echocardiography.

\section{Methods}

17 self-reported healthy volunteers were recruited under an IRB approved protocol. Ultra-fast phase contrast data was acquired on a $1.5 \mathrm{~T}$ Siemens Aera using both breath-hold (30 frames per cardiac cycle) and free breathing ( 50 frames per cardiac cycle) paradigms. To measure e' velocities, phase contrast data (Venc $25 \mathrm{~cm} / \mathrm{s}$ ) was acquired in the short axis orientation at a slice position where the myocardium on the apical side of the valve ring was within the slice throughout the cardiac cycle. To measure $\mathrm{E}$ and $\mathrm{A}$ velocities, phase contrast data $($ Venc $80 \mathrm{~cm} / \mathrm{s}$ ) was acquired in a single slice parallel to the mitral valve annulus, positioned such that the slice stayed below the valve throughout the entire cardiac cycle. E and A velocities as well as septal and lateral e' velocities were calculated using standard flow postprocessing. 2 subjects were excluded from both analyses due to improper gating, and 4 additional subjects were excluded from the breath hold analysis due to too much noise to identify e' velocities.

\section{Results}

With free breathing, E/A was measured at $1.7 \pm 0.5$ (range 0.8 - 2.6), septum E/e' was measured at $5.4 \pm 1.5$ (range $2.5-7.4$ ), and lateral E/e' was measured at $5.1 \pm$ 1.7 (range $2.6-8.4$ ). With breath hold, E/A was measured at $1.5 \pm 0.6$ (range $0.7-2.7$ ), septum E/e' was measured at $6.1 \pm 2.2$ (range $3.6-10.2$ ), and lateral E/e' was measured at $5.4 \pm 2.1$ (range 3.5 - 10.9). Subjects with E/e' above 8.0 had normal left atrial size.

\section{Conclusions}

Measured E/A and E/e' values are within normal limits using cutoff values that have been published with echocardiography [1], suggesting that phase contrast MRI may provide a complementary approach to assessing left ventricular diastolic function.

\section{Author details}

${ }^{1}$ Northwestern University Feinberg School of Medicine, Chicago, IL, USA.

${ }^{2}$ Northwestern University, Department of Radiology, Chicago, IL, USA.

${ }^{3}$ Siemens Medical Solutions, Chicago, IL, USA.

Published: 2 February 2011

\section{Reference}

1. Nagueh SF, Appleton CP, Gillebert TC, et al: Recommendations for the evaluation of left ventricular diastolic function by echocardiography. J Am Soc Echocardiogr 2009, 22:107.

doi:10.1186/1532-429X-13-S1-P237

Cite this article as: Chatterjee et al:: Phase contrast mri measurement of e/a and e/e'. Journal of Cardiovascular Magnetic Resonance 201113 (Suppl 1):P237. 